\title{
On Markov-Additive Jump Processes
}

\author{
Lothar Breuer ${ }^{\mathrm{a}}$ \\ ${ }^{a}$ FB IV - Computer Science, University of Trier, 54286 Trier, Germany \\ E-mail: breuer@info04.uni-trier.de
}

\begin{abstract}
In 1995, Pacheco and Prabhu introduced the class of so-called Markov-additive processes of arrivals in order to provide a general class of arrival processes for queueing theory. In this paper, the above class is generalized considerably, including time-inhomogeneous arrival rates, general phase spaces and the arrival space being a general vector space (instead of the finite-dimensional Euclidean space). Furthermore, the class of Markov-additive jump processes introduced in the present paper is embedded into the existing theory of jump processes. The best known special case is the class of BMAP arrival processes.
\end{abstract}

Keywords: jump processes, Markov-additive processes, queueing theory, BMAP

AMS Subject classification: 60J75, 60K25

\section{Introduction}

Inspired by a process developed in Ezhov, Skorokhod [10], the term "Markovadditive process" has been coined by Çinlar [6] in 1972. It refers to a two-dimensional Markov process with transition probabilities that depend on one dimension only. The marginal process in this dimension is a Markov process. The marginal process in the other dimension is a process with conditionally independent increments given the phase process.

The first idea of such a process goes back to Neveu [17] in 1961, whose so-called F-process is the class of Markov-additive processes with a finite phase space. A special case of F-processes is the class of BMAPs developed in Lucantoni [14] thirty years later, which are now widely used in queueing theory. The founding work of Çinlar [6] on Markov-additive processes was followed by several studies in the 1970s and 80s, e.g. Arjas, Speed [1], Çinlar [7,8,9], or Ney, Nummelin [18] for discrete time.

However, all these studies examined Markov-additive processes in the most general case. In the present paper, the focus is on Markov-additive processes which belong to the class of Markov jump processes. Thus it is possible to explore the many additional an- 
alytical opportunities which cannot be exploited in the general case of Markov-additive processes. The present paper mainly goes along the contribution by Pacheco, Prabhu [19], which was written after the BMAP concept proved successful as a versatile arrival process for queues. The concept developed in Pacheco, Prabhu [19] will be generalized in this paper and, most importantly, the inhomogeneous case will be analyzed, too.

In the present paper, the focus is on Markov-additive processes which belong to the class of Markov jump processes. As a reference for the theory of Markov jump processes, it will be referred to the introductory presentation in Gikhman, Skorokhod [11]. In the next section, these so-called Markov-additive jump processes are defined and the transition probabilities are derived in terms of the infinitesimal transition rates. Section 3 states some elementary properties, mostly resulting from the definition immediately. Under the assumption that the additive part of the state space is a real vector space, transforms and expectations are derived in section 4. The more specific assumption that the increments for the additive part be non-negative integers in every component of the vector space leads to the same derivations as in section 4 via the easier notion of $\mathrm{z}$ transforms. Since these processes represent the most general class of arrival processes for queueing theory, they will be called Markovian arrival processes (MAPs). MAPs are analyzed in section 5. In the last section, laws of large numbers are given for specific cases of Markov-additive jump processes on real vector spaces.

\section{Definition}

Markov-additive jump processes will be defined as two-dimensional Markov jump processes which satisfy the condition that the transition probabilities depend on one dimension only. The other dimension is an (additive) semi-group and the marginal process on it turns out to have conditionally independent increments.

Definition 1. Let $(N, J)=\left(\left(N_{t}, J_{t}\right): t \in \mathbb{R}_{0}^{+}\right)$be a two-dimensional Markov jump process with a locally compact and separable metric state space $S:=\Sigma \times \Phi$. Let $\sigma(\Sigma)$ and $\sigma(\Phi)$ be $\sigma$-algebras on $\Sigma$ and $\Phi$, respectively, which satisfy $\{x\} \in \sigma(\Sigma)$ and $\{y\} \in \sigma(\Phi)$ for all $x \in \Sigma$ and $y \in \Phi$. Denote $\mathcal{S}:=\sigma(\Sigma) \otimes \sigma(\Phi)$ as the product $\sigma$-algebra of $\sigma(\Sigma)$ and $\sigma(\Phi)$. Further, let $(\Sigma,+)$ be a semi-group with neutral element $0 \in \Sigma .(N, J)$ is called Markov-additive jump process if the transition probabilities satisfy the condition

$$
P(s, t ;(x, y), A \times B)=P(s, t ;(0, y),(A-x) \times B)
$$


for all $s<t \in \mathbb{R}_{0}^{+},(x, y) \in S$ and $A \times B \in \mathcal{S}$, with $A-x:=\{s \in \Sigma: s+x \in A\}$. Define

$$
P(s, t ; y, A \times B):=P(s, t ;(0, y), A \times B)
$$

for all $s<t \in \mathbb{R}_{0}^{+}, y \in \Phi$ and $A \times B \in \mathcal{S}$.

Remark 2. The above definition postulates that the state space $S:=\Sigma \times \Phi$ of a Markovadditive jump process be locally compact, separable and metric. Standard results in topology (cf. Herrlich [12], p.224,118,117) yield that $S$ satisfies this condition if and only if the state spaces $\Sigma$ and $\Phi$ of the marginal processes do so.

Remark 3. Because of equation 1, a Markov-additive jump process $(N, J)$ is uniquely determined by the probabilities $P(s, t ; y, A \times B)$. Since $(N, J)$ is a Markov jump process, the infinitesimal transition rates

$$
q(t ;(x, y), A \times B)=\lim _{h \rightarrow 0} \frac{P(t, t+h ;(x, y), A \times B)-1_{A \times B}(x, y)}{h}
$$

exist uniformly with respect to $(t,(x, y), A \times B)$ and the equality

$$
q(t ;(x, y), A \times B)=q(t ;(0, y),(A-x) \times B)
$$

follows from equation 1 . Hence, we can define

$$
q(t ; y, A \times B):=q(t ;(0, y), A \times B)
$$

for all $t \in \mathbb{R}_{0}^{+}, y \in \Phi$ and $A \times B \in \mathcal{S}$, and

$$
\begin{gathered}
\gamma(t, y):=-q(t ; y,\{(0, y)\}) \\
\gamma(t, y, A \times B):=q(t ; y,(A \times B) \backslash\{(0, y)\})
\end{gathered}
$$

as well as

$$
p(t, y, A \times B):=\left\{\begin{array}{cll}
\frac{\gamma(t, y, A \times B)}{\gamma(t, y)} & \text { for } & \gamma(t, y)>0 \\
1_{A \times B}(0, y) & \text { for } & \gamma(t, y)=0
\end{array}\right.
$$

Finally, define the kernel $Q(t)$ on $\Sigma \times \Phi$ by

$$
Q(t ;(x, y), A \times B):=q(t ; y,(A-x) \times B)
$$

for all $t \in \mathbb{R}_{0}^{+},(x, y) \in S$ and $A \times B \in \mathcal{S}$. The kernel $Q(t)$ is called the generator of $(N, J)$ at time $t$. 
Remark 4. By definition, a Markov-additive jump process is translation invariant or homogeneous in the first component. This leads to a self-similar structure of the generators $\left(Q(t): t \in \mathbb{R}_{0}^{+}\right)$, which can be illustrated in the case of an homogeneous Markovadditive jump process with $\Phi$ being finite and $\Sigma=I N_{0}$ (the so-called Batch Markovian Arrival Process or BMAP, see Lucantoni [14]). Here, the generator $Q$, which is constant in time, takes the form

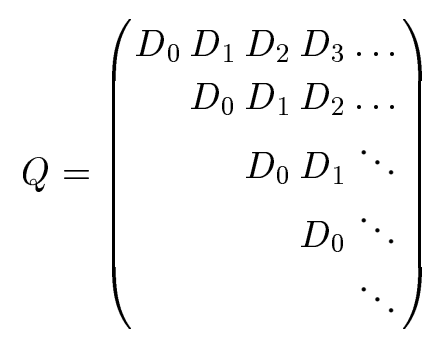

with $m \times m$-matrices $\left(D_{n}: n \in I N_{0}\right), m$ being the size of $\Phi$. Omitting the first row and first column, one obtains the matrix $Q$ again. This self-similarity yields many simplifications for the analysis of queues with BMAP arrivals. For a so-called leveldependent BMAP, which does not possess this self-similarity, and for the analysis of queues with level-dependent BMAP arrivals see Hofmann [13].

Remark 5. An example of a Markov-additive jump process with an additive space $\Sigma$ of infinite dimension is the class of Spatial Markovian Arrival Processes (SMAPs, see Breuer [5]). This process is used in the modelling of mobile communication networks, for which the location of users is important for the performance analysis of the network. For SMAPs, a so-called arrival space $R$ with measurable subsets $S \in \sigma(R)$ is defined to represent the area in which users of the network may appear and move in. An SMAP is a Markov-additive jump process with $\Sigma$ being the measurable space of counting functions on $(R, \sigma(R))$. This is a subset of the vector space $I N_{0}^{\sigma(R)}$. Thus an SMAP is a special Markovian arrival process which will be defined and further examined in section 5 .

The transition probabilities of the process will be derived as the solutions of the Kolmogorov differential equations via the method of successive approximations by $\mathrm{Pi}$ card and Lindelöf. Let $(N, J)$ be a Markov-additive jump process. Then the Kolmogorov differential equations take the following form for $(N, J)$ :

$$
\frac{\partial P(s, t ; y, A \times B)}{\partial t}=\int_{\Sigma \times \Phi} q(t ; w,(A-v) \times B) P(s, t ; y, d(v, w))
$$


for all $t>s$ (Kolmogorov's forward equation) and

$$
\frac{\partial P(s, t ; y, A \times B)}{\partial s}=-\int_{\Sigma \times \Phi} P(s, t ; w,(A-v) \times B) q(s ; y, d(v, w))
$$

for all $s<t$ (Kolmogorov's backward equation). Both differential equations contain a convolution in the first dimension. This is a consequence of the additivity which is defined on the marginal state space $\Sigma$. The convolution form will be preserved in the transition probabilities, as the next theorems show.

Theorem 6. The transition probabilities of a Markov-additive jump process can also be expressed in the form

$$
P(s, t ; y, A \times B)=\sum_{n=0}^{\infty} P^{(n)}(s, t ; y, A \times B)
$$

with

$$
P^{(0)}(s, t ; y, A \times B):=1_{A \times B}(0, y)
$$

and recursively

$$
P^{(n+1)}(s, t ; y, A \times B):=\int_{s}^{t} \int_{\Sigma \times \Phi} P^{(n)}(s, u ; y, d(v, w)) q(u ; w,(A-v) \times B) d u
$$

for all $n \in I N_{0}$.

Proof: By definition, the infinitesimal rates $q(t ; x, A)$ are continuous in $t$ for every $x \in S$ and $A \in \mathcal{S}$. Induction by $n$ yields that $P^{(n)}(s, t ; x, A)$ is differentiable in $t$ for every $n \in \mathbb{I N}_{0}$. Now, direct validation shows

$$
\begin{aligned}
\frac{\partial}{\partial t} \sum_{n=0}^{\infty} P^{(n)}(s, t ; x, A) & =\frac{\partial}{\partial t} \sum_{n=1}^{\infty} P^{(n)}(s, t ; x, A) \\
& =\sum_{n=1}^{\infty} \frac{\partial}{\partial t} \int_{s}^{t} \int_{S} P^{(n-1)}(s, u ; x, d y) q(u ; y, A) d u \\
& =\sum_{n=1}^{\infty} \int_{S} P^{(n-1)}(s, t ; x, d y) q(t ; y, A) \\
& =\int_{S} q(t ; y, A) \sum_{n=0}^{\infty} P^{(n)}(s, t ; x, d y)
\end{aligned}
$$

i.e. the Kolmogorov forward equation is satisfied. According to Gikhman, Skorokhod [11], p.317 (theorem 3), the solution to the Kolmogorov forward equation is unique.

() 
By induction, one can prove that

$$
P^{(n)}(s, t ; y, A \times B)=\underbrace{\int_{s}^{t} \int_{s}^{u_{n}} \ldots \int_{s}^{u_{2}}}_{n \text { integrals }}\left(Q\left(u_{1}\right) \ldots Q\left(u_{n}\right)\right)((0, y), A \times B) d u_{1} \ldots d u_{n}
$$

with $Q(u)$ denoting the generator at time $u \in[s, t]$. An iteration for computing the transition probabilities is given by starting with

$$
P_{0}(s, t ; y, A \times B):=1_{A \times B}(0, y)
$$

and iterating by

$P_{n+1}(s, t ; y, A \times B):=\int_{s}^{t} \int_{S} P_{n}(s, u ; y, d(v, w)) q(u ; w,(A-v) \times B) d u+1_{A \times B}(0, y)$

for all $n \in I N_{0}$.

\section{Elementary Properties}

Some elementary properties for Markov-additive jump processes can be taken from Çinlar [6] and Ezhov, Skorokhod [10], who examined the more general class of Markov-additive processes. This section contains some properties which mostly are immediate consequences of the definition 1. Obviously, the basic sample path properties of Markov jump processes hold for the subclass of Markov-additive jump processes, too. Furthermore, the distribution of the holding time in a state of the additive space $\Sigma$ can be given. An immediate result is

Theorem 7. Let $(N, J)$ be a Markov-additive jump process with state space $\Sigma \times \Phi$. Then the marginal process $J$ is a Markov jump process with state space $\Phi$ and transition probabilities

$$
P^{\Phi}(s, t ; y, B)=P(s, t ; y, \Sigma \times B)
$$

for all $s<t \in \mathbb{R}_{0}^{+}, y \in \Phi$ and $B \in \sigma(\Phi)$.

Remark 8. This theorem gives rise to a method of analysis which regards the marginal process $J$ as the independent underlying Markov jump process that conditions the additive process $N$. The marginal state space $\Phi$ shall be called phase space of $(N, J)$, a single element of $\Phi$ is called a phase. The marginal process $J$ shall be called phase process of $(N, J)$, while $N$ shall be called additive process of $(N, J)$. The next theorem 
shows that $N$ has conditionally independent increments given $J$. Furthermore, theorem 11 yields a representation of the generator as the product of two kernels.

Theorem 9. Let $(N, J)$ be a Markov-additive jump process with state space $\Sigma \times \Phi$. Define $T:=\inf \left\{t \in \mathbb{R}^{+}: N_{t} \neq N_{0}\right\}$ as the holding time of the marginal process $N$ in a state of $\Sigma$. Further, define the sub-Markov kernel $P(T>t)$ on $\Phi$ by

$$
P(T>t)(y, B):=P\left(T>t, J_{t} \in B \mid J_{0}=y\right)
$$

for all $t \in \mathbb{R}^{+}, y \in \Phi$ and $B \in \sigma(\Phi)$. Then for all $s, t \in \mathbb{R}^{+}$,

$$
P(T>t+s)=P(T>t) P(T>s)
$$

and furthermore,

$$
P(T>t)(y, B)=\sum_{n=0}^{\infty} \underbrace{\int_{0}^{t} \int_{0}^{u_{n}} \ldots \int_{0}^{u_{2}}}_{n \text { integrals }}\left(Q_{0}\left(u_{1}\right) \ldots Q_{0}\left(u_{n}\right)\right)(y, B) d u_{1} \ldots d u_{n}
$$

with

$$
Q_{0}(t)(y, B):=Q(t ; y,\{0\} \times B)
$$

for all $t \in \mathbb{R}_{0}^{+}, y \in \Phi$ and $B \in \sigma(\Phi)$.

Proof: The first equation follows from the definition of $P(T>t)$ and the fact that $(N, J)$ is a Markov-additive jump process. For the second equation, note that the holding time $T$ is identical to the first hitting time $T_{A}$ of the set $A:=\Sigma \backslash\{0\} \times \Phi$. This equals the life time of the cut-off Markov jump process with state space $\Phi$ and generator $\left(Q_{0}(t): t \in \mathbb{R}_{0}^{+}\right)$. Then statement 5 is merely the form for the transition probability kernel of the cut-off process, which follows from theorem 6. (-)

Remark 10. This result contains the statements of theorems 6.9 and 6.11 in Pacheco, Prabhu [19] as the special case of $(N, J)$ being homogeneous, $\Sigma=I N_{0}^{r}$ and $\Phi$ being countable.

For later use, a fundamental theorem will be stated which asserts the existence of a representation of the infinitesimal transition rates (resp. the transition probabilities) as the product of a kernel on the phase space $\Phi$ (which is a function $\Phi \times \sigma(\Phi) \rightarrow[0,1]$ ) and a kernel from $\Phi$ into the additive space $\Sigma$ (which is a function $\Phi \times \sigma(\Sigma) \rightarrow[0,1]$ ). 
Theorem 11. For every $t \in \mathbb{R}_{0}^{+}$and $y \in \Phi$, there is a kernel $K_{t, y}: \Phi \times \sigma(\Sigma) \rightarrow \mathbb{R}$ such that

$$
Q(t ; y, A \times B)=\int_{B} Q(t ; y, \Sigma \times d z) K_{t, y}(z, A)
$$

for all $A \in \sigma(\Sigma)$ and $B \in \sigma(\Phi)$. Furthermore, for every $s<t \in \mathbb{R}_{0}^{+}$and $y \in \Phi$, there is a kernel $L_{s, t, y}: \Phi \times \sigma(\Sigma) \rightarrow \mathbb{R}$ such that

$$
P(s, t ; y, A \times B)=\int_{B} P(s, t ; y, \Sigma \times d z) L_{s, t, y}(z, A)
$$

for all $A \in \sigma(\Sigma)$ and $B \in \sigma(\Phi)$. The kernels $K_{t, y}$ and $L_{s, t, y}$ are almost surely uniquely determined.

Proof: See Bauer [2], p.397 (with $\mathcal{C}=\Sigma \times \sigma(\Phi)$ ), or Bourbaki [4], p.39 (with $p=p r_{2}$ ), since the charge $Q(t ; y,$.$) can be represented as the difference of two finite measures.$

\section{Markov-Additive Jump Processes on a Real Vector Space}

Now assume that $(\Sigma,+)$ is a real vector space. Then $(\Sigma,+)$ has a base $\left(b_{i}: i \in I\right)$ with some index set $I$, and every element $x \in \Sigma$ has a unique representation $\left(c_{i}: i \in I\right)$ in $\mathbb{R}^{I}$ with respect to this base (cf. Nef [16], p.46). Denote the bijective mapping between an element $x \in \Sigma$ and its representation $\left(c_{i}: i \in I\right)$ by $f: \Sigma \rightarrow \mathbb{R}^{I}$. In order to simplify the notation in the following, an element $x \in \Sigma$ and its representation $f(x) \in \mathbb{R}^{I}$ shall be identified. Further, denote the projection from $\mathbb{R}^{I}$ to the $i$-th component by $p r_{i}: \mathbb{R}^{I} \rightarrow \mathbb{R}$.

Under this assumption, transforms as well as expectations can be derived for the marginal process $N$ of a Markov-additive jump processes $(N, J)$. Using the structure of the vector space, we can define the expectation of a Markov-additive jump processes with state space $\Sigma \times \Phi$ as follows:

Definition 12. Let $(N, J)$ be a Markov-additive jump process with state space $\Sigma \times \Phi$ and $(\Sigma,+)$ be a real vector space. The expectation $E\left(N_{t}-N_{s}\right)$ of the marginal process $N$ during the time interval $] s, t]$ is defined as the kernel on $\Phi$ with entries

$$
E\left(N_{t}-N_{s}\right)(y, B):=\left(E\left(p r_{i}\left(N_{t}-N_{s}\right) \cdot 1_{B}\left(J_{t}\right) \mid J_{s}=y\right): i \in I\right)
$$

For real valued random variables, the Fourier transform has proved very useful, especially for determining moments (cf. Bauer [2], pp.183-223). The same analytical method shall be applied here for every dimension of the vector space $\Sigma$. 
Definition 13. Let $(N, J)$ be a Markov-additive jump process with state space $\Sigma \times \Phi$ and $(\Sigma,+)$ be a real vector space. Then the Fourier transform $N_{s, t}^{*}$ of $(N, J)$ over the time interval $] s, t]$ shall be defined as the function $r \rightarrow N_{s, t}^{*}(r)$ with

$$
N_{s, t}^{*}(r)(y, B):=\left(\int_{-\infty}^{\infty} e^{i \cdot r c} P\left(s, t ; y, p r_{j}^{-1}(d c) \times B\right): j \in I\right)
$$

for all $r \in \mathbb{R}, y \in \Phi$ and $B \in \sigma(\Phi)$, with $i$ denoting the imaginary unit in the space $C$ of complex numbers.

Remark 14. Note that by theorem 11 , the distribution $P\left(s, t ; y, p r_{j}^{-1}(d c) \times B\right)$ can be written as

$$
P\left(s, t ; y, p r_{j}^{-1}(d c) \times B\right)=\int_{B} P(s, t ; y, \Sigma \times d z) L_{s, t, y}(z, d c)
$$

with some kernel $L_{s, t, y}$ from $\Phi$ to $\Sigma$. Hence the representation

$$
p r_{j}\left(N_{s, t}^{*}(r)(y, B)\right):=\int_{B} \int_{-\infty}^{\infty} e^{i \cdot r c} L_{s, t, y}(z, d c) P(s, t ; y, \Sigma \times d z)
$$

holds and thus every component $\operatorname{pr}_{j}\left(N_{s, t}^{*}(r)\right)$ is a kernel on $\Phi$.

Theorem 15. For every $j \in I, y \in \Phi, B \in \sigma(\Phi)$ and $t \in \mathbb{R}_{0}^{+}$, define the kernel $\varphi_{Q^{(j)}(t)}(r)$ on $\Phi$ by its entries

$$
\begin{aligned}
\varphi_{Q^{(j)}(t)}(r)(y, B) & :=\int_{-\infty}^{\infty} e^{i \cdot r c} Q\left(t ; y, p r_{j}^{-1}(d c) \times B\right) \\
& =\int_{B} \int_{-\infty}^{\infty} e^{i \cdot r c} K_{t, y}\left(z, p r_{j}^{-1}(d c)\right) Q(t ; y, \Sigma \times d z)
\end{aligned}
$$

with some kernel $K_{t, y}$ from $\Phi$ to $\Sigma$ (see theorem 11 for the second equality). Then the Fourier transform of $(N, J)$ over the time interval $] s, t]$ can be written as

$$
N_{s, t}^{*}(r)=(\sum_{n=0}^{\infty} \underbrace{\int_{s}^{t} \int_{s}^{u_{n}} \ldots \int_{s}^{u_{2}}}_{n \text { integrals }} \varphi_{Q^{(j)}\left(u_{1}\right)}(r) \ldots \varphi_{Q^{(j)}\left(u_{n}\right)}(r) d u_{1} \ldots d u_{n}: j \in I)
$$

for all $r \in \mathbb{R}$, with the summand for $n=0$ being the identity kernel $I d$ on $\Phi$. 
Proof: Fix $i \in I$ as well as $y \in \Phi$ and $B \in \sigma(\Phi)$. According to formula 3, the $j$ th component of $N_{s, t}^{*}(r)(y, B)$ equals

$$
\begin{aligned}
& p r_{j}\left(N_{s, t}^{*}(r)(y, B)\right)=\sum_{n=0}^{\infty} \int_{-\infty}^{\infty} e^{i \cdot r c} P^{(n)}\left(s, t ; y, p r_{j}^{-1}(d c) \times B\right) \\
= & \sum_{n=0}^{\infty} \underbrace{\int_{s}^{t} \int_{s}^{u_{n}} \ldots \int_{s}^{u_{2}}}_{n \text { integrals }} \int_{-\infty}^{\infty} e^{i \cdot r c}\left(Q\left(u_{1}\right) \ldots Q\left(u_{n}\right)\right)\left((0, y), p r_{j}^{-1}(d c) \times B\right) \\
& d u_{1} \ldots d u_{n} \\
= & \sum_{n=0}^{\infty} \int_{s}^{t} \int_{s}^{u_{n}} \ldots \int_{s}^{u_{2}} \int_{\mathbb{R} \times \Phi} Q\left(u_{1} ; y, d\left(c_{1}, y_{1}\right)\right) e^{i \cdot r c_{1}} \ldots \\
& \ldots \int_{\mathbb{R} \times \Phi} Q\left(u_{n-1} ; y_{n-2}, d\left(c_{n-1}, y_{n-1}\right)\right) e^{i \cdot r c_{n-1}} \int_{\mathbb{R} \times B} Q\left(u_{n} ; y_{n-1}, d\left(c_{n}, y_{n}\right)\right) e^{i \cdot r c_{n}} \\
& d u_{1} \ldots d u_{n}
\end{aligned}
$$

by writing out the convolution $c=c_{1}+\ldots+c_{n}$ explicitly. Further, application of theorem 11 leads to

$$
\begin{aligned}
p_{j} & \left(N_{s, t}^{*}(r)(y, B)\right)= \\
= & \left.\sum_{n=0}^{\infty} \int_{s}^{t} \int_{s}^{u_{n}} \ldots \int_{s}^{u_{2}} \int_{\Phi} \int_{-\infty}^{\infty} e^{i \cdot r c_{1}} K_{u_{1}, y}\left(y_{1}, p r_{j}^{-1}\left(d c_{1}\right)\right) Q\left(u_{1} ; y, \Sigma \times d y_{1}\right)\right) \ldots \\
& \ldots \int_{\Phi} \int_{-\infty}^{\infty} e^{i \cdot r c_{n-1}} K_{u_{n-1}, y_{n-2}}\left(y_{n-1}, p r_{j}^{-1}\left(d c_{n-1}\right)\right) Q\left(u_{n-1} ; y_{n-2}, \Sigma \times d y_{n-1}\right) \\
& \int_{B} \int_{-\infty}^{\infty} e^{i \cdot r c_{n}} K_{u_{n}, y_{n-1}}\left(y_{n}, p r_{j}^{-1}\left(d c_{n}\right)\right) Q\left(u_{n} ; y_{n-1}, \Sigma \times d y_{n}\right) d u_{1} \ldots d u_{n} \\
= & \sum_{n=0}^{\infty} \int_{s}^{t} \int_{s}^{u_{n}} \ldots \int_{s}^{u_{2}} \int_{\Phi} \varphi_{Q^{(j)}\left(u_{1}\right)}(r)\left(y, d y_{1}\right) \ldots \int_{B} \varphi_{Q^{(j)}\left(u_{n}\right)}(r)\left(y_{n-1}, d y_{n}\right) \\
& d u_{1} \ldots d u_{n} \\
= & \sum_{n=0}^{\infty} \int_{s}^{t} \int_{s}^{u_{n}} \ldots \int_{s}^{u_{2}}\left(\varphi_{Q^{(j)}\left(u_{1}\right)}(r) \ldots \varphi_{Q^{(j)}\left(u_{n}\right)}(r)\right)(y, B) d u_{1} \ldots d u_{n}
\end{aligned}
$$

Theorem 16. Define the kernel $M^{(j)}(t)$ on $\Phi$ by

$$
M^{(j)}(t)(y, B):=\int_{-\infty}^{\infty} c Q\left(t ; y, p r_{j}^{-1}(d c) \times B\right)
$$


for all $t \in \mathbb{R}, y \in \Phi$ and $B \in \sigma(\Phi)$. Then the expectation of the marginal process $N$ over the time interval $] s, t]$ is given by

$$
E\left(N_{t}-N_{s}\right)=\left(\int_{s}^{t} P^{\Phi}(s, u) M^{(j)}(u) P^{\Phi}(u, t) d u: j \in I\right)
$$

Proof: The expectation $E\left(N_{t}-N_{s}\right)$ can be computed as

$$
E\left(N_{t}-N_{s}\right)=-\left.i \cdot \frac{d}{d r} N_{s, t}^{*}(r)\right|_{r=0}
$$

recognizing that the differentiation can be performed component-wise. Then the $j$ th component of $E\left(N_{t}-N_{s}\right)$ is

$$
\begin{aligned}
& \operatorname{pr}_{j}\left(E\left(N_{t}-N_{s}\right)\right)= \\
& \quad=-\left.i \cdot \sum_{n=1}^{\infty} \underbrace{\int_{s}^{t} \int_{s}^{u_{n}} \ldots \int_{s}^{u_{2}}}_{n \text { integrals }} \frac{d}{d r}\left(\varphi_{Q^{(j)}\left(u_{1}\right)}(r) \ldots \varphi_{Q^{(j)}\left(u_{n}\right)}(r)\right)\right|_{r=0} d u_{1} \ldots d u_{n}
\end{aligned}
$$

according to the preceding theorem 15. Applying the product rule of differentiation, this equals

$$
\begin{aligned}
p r_{j}( & \left.E\left(N_{t}-N_{s}\right)\right)= \\
= & -i \cdot \sum_{n=1}^{\infty} \underbrace{\int_{s}^{t} \int_{s}^{u_{n}} \ldots \int_{s}^{u_{2}} \sum_{l=1}^{n} \varphi_{Q^{(j)}\left(u_{1}\right)}(0) \ldots \varphi_{Q^{(j)}\left(u_{l-1}\right)}(0)}_{n \text { integrals }} \\
& \left(\left.\frac{d}{d r} \varphi_{Q^{(j)}\left(u_{l}\right)}(r)\right|_{r=0}\right) \varphi_{Q^{(j)}\left(u_{l+1}\right)}(0) \ldots \varphi_{Q^{(j)}\left(u_{n}\right)}(0) d u_{1} \ldots d u_{n} \\
= & \sum_{l=1}^{\infty} \sum_{n=l}^{\infty} \underbrace{\int_{s}^{t} \int_{s}^{u_{n}} \ldots \int_{s}^{u_{l+2}}}_{n-l \text { integrals }} \int_{s}^{u_{l+1}} \\
& \underbrace{\int_{s}^{u_{l}} \ldots \int_{s}^{u_{2}} \varphi_{Q^{(j)}\left(u_{1}\right)}(0) \ldots \varphi_{Q^{(j)}\left(u_{l-1}\right)}(0) d u_{1} \ldots d u_{l-1}}_{l-1 \text { integrals }} \\
& \left(-\left.i \cdot \frac{d}{d r} \varphi_{Q^{(j)}\left(u_{l}\right)}(r)\right|_{r=0}\right) d u_{l} \varphi_{Q^{(j)}\left(u_{l+1}\right)}(0) \ldots \varphi_{Q^{(j)}\left(u_{n}\right)}(0) d u_{l+1} \ldots d u_{n}
\end{aligned}
$$

Acknowledging that

$$
-\left.i \cdot \frac{d}{d r} \varphi_{Q^{(j)}\left(u_{l}\right)}(r)\right|_{r=0}(y, B)=\int_{-\infty}^{\infty} c Q\left(u_{l} ; y, p r_{j}^{-1}(d c) \times B\right)=M^{(j)}\left(u_{l}\right)(y, B)
$$


is the expectation kernel of the $j$ th component and

$$
\varphi_{Q^{(j)}(u)}(0)(y, B)=Q(u ; y, \Sigma \times B)
$$

is the infinitesimal transition rate of the phase process leads to

$$
\begin{aligned}
p_{j}( & \left.E\left(N_{t}-N_{s}\right)\right)= \\
= & \int_{s}^{t}(\sum_{l=1}^{\infty} \underbrace{\int_{s}^{u_{l}} \ldots \int_{s}^{u_{2}}}_{l-1 \text { integrals }} \varphi_{Q^{(j)}\left(u_{1}\right)}(0) \ldots \varphi_{Q^{(j)}\left(u_{l-1}\right)}(0) d u_{1} \ldots d u_{l-1}) M^{(j)}\left(u_{l}\right) \\
& (\sum_{n=l}^{\infty} \underbrace{\int_{u_{l}}^{t} \int_{u_{l}}^{u_{n}} \ldots \int_{u_{l}}^{u_{l+2}}}_{n-l \text { integrals }} \varphi_{Q^{(j)}\left(u_{l+1}\right)}(0) \ldots \varphi_{Q^{(j)}\left(u_{n}\right)}(0) d u_{l+1} \ldots d u_{n}) d u_{l} \\
= & \int_{s}^{t} P^{\Phi}\left(s, u_{l}\right) M^{(j)}\left(u_{l}\right) P^{\Phi}\left(u_{l}, t\right) d u_{l}
\end{aligned}
$$

(-)

\section{Markovian Arrival Processes}

In queueing theory, the most important notion is that of the number of users or customers in the system (which is the queue or the queueing network). The values of this are limited to the set $I N_{0}$ of non-negative integers. Thus for most applications in queueing theory, the use of Markov-additive processes $(N, J)$ on real vector spaces (of dimension $|I|)$ with $I N_{0}^{I}$-valued marginal process $N$ suffices.

If the support of the marginal process $N$ is on $I N_{0}^{I}$, i.e. if $P\left(p r_{i}\left(N_{t}\right) \in I N_{0}\right)=1$ for all $i \in I$ and $t \in \mathbb{R}_{0}^{+}$, then the easier notion of a z-transform serves the same purposes as the Fourier transform. In this section, the same analysis as in the preceding section 4 shall be carried out using z-transforms.

Definition 17. Let $(N, J)$ be a Markov-additive jump process with state space $\Sigma \times \Phi$ and $(\Sigma,+)$ be a real vector space having a base $\left(b_{i}: i \in I\right)$. If the marginal process $N$ has support $I N_{0}^{I}$, then $(N, J)$ shall be called Markovian arrival process. 
Remark 18. The homogeneity of the first component implies that Markovian arrival processes have only non-negative increments in the first dimension. This follows from property 1 , since for every $n \in \Sigma, k \in \mathbb{I N}$ and any dimension $i \in I$ of the vector space,

$$
P\left(s, t ;(n, y), p r_{i}^{-1}\left(p r_{i}(n)-k\right) \times B\right)=P\left(s, t ; y, p r_{i}^{-1}(-k) \times B\right)=0
$$

because of $p r_{i}^{-1}(-k) \notin I N_{0}^{I}$.

Definition 19. Let $(N, J)$ be a Markovian arrival process. Define the z-transform of $(N, J)$ over the time interval $] s, t]$ as the function $z \rightarrow N(s, t ; z)$ with values being the kernels on $\Phi$ which are determined by

$$
N(s, t ; z)(y, B):=\left(\sum_{n=0}^{\infty} P\left(p r_{i}\left(N_{t}-N_{s}\right)=n, J_{t} \in B \mid J_{s}=y\right) z^{n}: i \in I\right)
$$

for all $z \in C$ with $|z| \leq 1, y \in \Phi$ and $B \in \sigma(\Phi)$.

Theorem 20. For every $i \in I, k \in I N_{0}$ and $t \in \mathbb{R}_{0}^{+}$, define the kernels $Q_{k}^{(i)}(t)$ on $\Phi$ by

$$
Q_{k}^{(i)}(t)(y, B):=Q\left(t ; y, p r_{i}^{-1}(k) \times B\right)
$$

for all $y \in \Phi$ and $B \in \sigma(\Phi)$. Then the z-transform of $(N, J)$ over the time interval $] s, t]$ can be written as

$$
\begin{aligned}
& N(s, t ; z)= \\
& =(\sum_{n=0}^{\infty} \underbrace{\int_{s}^{t} \int_{s}^{u_{n}} \ldots \int_{s}^{u_{2}}}_{n \text { integrals }} \sum_{k=0}^{\infty} Q_{k}^{(i)}\left(u_{1}\right) z^{k} \ldots \sum_{k=0}^{\infty} Q_{k}^{(i)}\left(u_{n}\right) z^{k} d u_{1} \ldots d u_{n}: i \in I)
\end{aligned}
$$

for all $z \in C$ with $|z| \leq 1$. 
Proof: Fix $i \in I$ as well as $y \in \Phi$ and $B \in \sigma(\Phi)$. According to formula 3, the $i$ th component of $N(s, t ; z)(y, B)$ equals

$$
\begin{aligned}
& N(s, t ; z)(y, B)=\sum_{k=0}^{\infty} \sum_{n=0}^{\infty} P^{(n)}\left(s, t ; y, p r_{i}^{-1}(k) \times B\right) \cdot z^{k} \\
& =\sum_{k=0}^{\infty} \sum_{n=0}^{\infty} \underbrace{\int_{s}^{t} \int_{s}^{u_{n}} \ldots \int_{s}^{u_{2}}}_{n \text { integrals }}\left(Q\left(u_{1}\right) \ldots Q\left(u_{n}\right)\right)\left((0, y), p r_{i}^{-1}(k) \times B\right) d u_{1} \ldots d u_{n} \cdot z^{k} \\
& =\sum_{n=0}^{\infty} \underbrace{\int_{s}^{t} \int_{s}^{u_{n}} \ldots \int_{s}^{u_{2}}}_{n \text { integrals }} \sum_{k=0}^{\infty} \sum_{k_{1}+\ldots+k_{n}=k}\left(Q_{k_{1}}^{(i)}\left(u_{1}\right) \ldots Q_{k_{n}}^{(i)}\left(u_{n}\right)\right)(y, B) \cdot z^{k} d u_{1} \ldots d u_{n} \\
& =\sum_{n=0}^{\infty} \underbrace{\int_{s}^{t} \int_{s}^{u_{n}} \ldots \int_{s}^{u_{2}}}_{n \text { integrals }}\left(\sum_{k=0}^{\infty} Q_{k}^{(i)}\left(u_{1}\right) z^{k} \ldots \sum_{k=0}^{\infty} Q_{k}^{(i)}\left(u_{n}\right) z^{k}\right)(y, B) d u_{1} \ldots d u_{n}
\end{aligned}
$$

since the z-transform of the convolution of the kernels $Q_{k_{1}}^{(i)}\left(u_{1},\right) \ldots, Q_{k_{n}}^{(i)}\left(u_{n}\right)$ equals the product of the z-transforms of $Q_{k_{1}}^{(i)}\left(u_{1}\right), \ldots, Q_{k_{n}}^{(i)}\left(u_{n}\right)$.

())

Remark 21. Note that $\sum_{k=0}^{\infty} Q_{k}^{(i)}\left(u_{j}\right) z^{k}$ is a kernel on $\Phi$ for every $j \in I N$ and thus every product $\sum_{k=0}^{\infty} Q_{k}^{(i)}\left(u_{1}\right) z^{k} \ldots \sum_{k=0}^{\infty} Q_{k}^{(i)}\left(u_{n}\right) z^{k}$ is a kernel on $\Phi$, too.

Theorem 22. The expectation of the marginal process $N$ over the time interval $] s, t]$ is given by

$$
E\left(N_{t}-N_{s}\right)=\left(\int_{s}^{t} P^{\Phi}(s, u) \sum_{k=1}^{\infty} k \cdot Q_{k}^{(i)}(u) P^{\Phi}(u, t) d u: i \in I\right)
$$

Proof: The expectation $E\left(N_{t}-N_{s}\right)$ can be computed as

$$
E\left(N_{t}-N_{s}\right)=\left.\frac{d}{d z} N(s, t ; z)\right|_{z=1}
$$

recognizing that the differentiation can be performed component-wise. Then the $i$ th component of $E\left(N_{t}\right)$ is

$$
\begin{aligned}
& \operatorname{pr}_{i}\left(E\left(N_{t}-N_{s}\right)\right)= \\
& =\left.\sum_{n=1}^{\infty} \underbrace{\int_{s}^{t} \int_{s}^{u_{n}} \ldots \int_{s}^{u_{2}}}_{n \text { integrals }} \frac{d}{d z}\left(\sum_{k=0}^{\infty} Q_{k}^{(i)}\left(u_{1}\right) z^{k} \ldots \sum_{k=0}^{\infty} Q_{k}^{(i)}\left(u_{n}\right) z^{k}\right)\right|_{z=1} d u_{1} \ldots d u_{n}
\end{aligned}
$$


Denote the generator of the phase process at time $u$ by $Q^{\Phi}(u)$ and note that

$$
Q^{\Phi}(u)=\sum_{k=0}^{\infty} Q_{k}^{(i)}(u)
$$

is independent of $i \in I$. Applying the product rule of differentiation yields

$$
\begin{aligned}
& p r_{i}\left(E\left(N_{t}-N_{s}\right)\right)= \\
& =\sum_{n=1}^{\infty} \underbrace{\int_{s}^{t} \int_{s}^{u_{n}} \ldots \int_{s}^{u_{2}}}_{n \text { integrals }} \sum_{l=1}^{n} Q^{\Phi}\left(u_{1}\right) \ldots Q^{\Phi}\left(u_{l-1}\right)\left(\sum_{k=1}^{\infty} k Q_{k}^{(i)}\left(u_{l}\right)\right) Q^{\Phi}\left(u_{l+1}\right) \ldots \\
& \ldots Q^{\Phi}\left(u_{n}\right) d u_{1} \ldots d u_{n}
\end{aligned}
$$

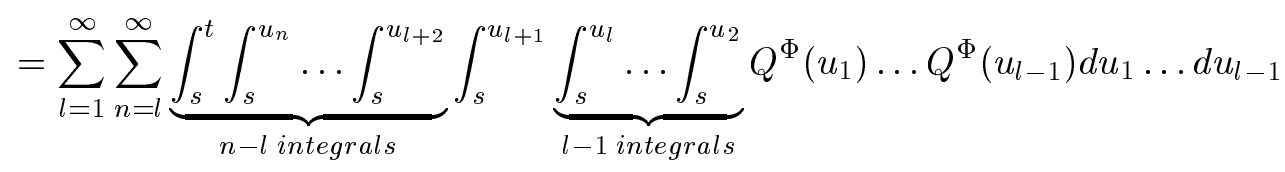

$$
\begin{aligned}
& \left(\sum_{k=1}^{\infty} k Q_{k}^{(i)}\left(u_{l}\right)\right) d u_{l} Q^{\Phi}\left(u_{l+1}\right) \ldots Q^{\Phi}\left(u_{n}\right) d u_{l+1} \ldots d u_{n} \\
& =\int_{s}^{t}(\sum_{l=1}^{\infty} \underbrace{\int_{s}^{u_{l}} \ldots \int_{s}^{u_{2}}}_{l-1 \text { integrals }} Q^{\Phi}\left(u_{1}\right) \ldots Q^{\Phi}\left(u_{l-1}\right) d u_{1} \ldots d u_{l-1}) \sum_{k=1}^{\infty} k Q_{k}^{(i)}\left(u_{l}\right) \\
& (\sum_{n=l}^{\infty} \underbrace{\int_{u_{l}}^{t} \int_{u_{l}}^{u_{n}} \ldots \int_{u_{l}}^{u_{l+2}}}_{n-l \text { integrals }} Q^{\Phi}\left(u_{l+1}\right) \ldots Q^{\Phi}\left(u_{n}\right) d u_{l+1} \ldots d u_{n}) d u_{l} \\
& =\int_{s}^{t} P^{\Phi}\left(s, u_{l}\right)\left(\sum_{k=1}^{\infty} k Q_{k}^{(i)}\left(u_{l}\right)\right) P^{\Phi}\left(u_{l}, t\right) d u_{l}
\end{aligned}
$$

remembering that the transition probabilities of the phase process are denoted by $P^{\Phi}(s, t)$

(:)

Remark 23. The first moment formulae given in Pacheco, Prabhu [19], theorem 6.15 and corollary 6.16(a), can be obtained from this theorem as the special case of $(N, J)$ being homogeneous, $\Sigma=I N_{0}^{r}$ and $\Phi$ being countable. 


\section{Laws of Large Numbers}

For some special cases of Markov-additive jump processes on real vector spaces, the asymptotic behaviour can be described in terms of strong laws of large numbers. First, this will be done for processes with periodic generators. The case of homogeneous processes shall turn out to be a corollary of the periodic case. In this section, convergence on the vector space $\Sigma$ shall be defined in a weak sense as convergence in every dimension of $\Sigma$.

Definition 24. A Markov-additive jump process $(N, J)$ with time-dependent generator $\left(Q(t): t \in \mathbb{R}_{0}^{+}\right)$is called periodic with period $T>0$ if $Q(s+T)=Q(s)$ for every $s \in\left[0, T\left[\right.\right.$. A family $\left(q_{s}: s \in[0, T[)\right.$ of probability distributions shall be called a periodic family of asymptotic distributions for the phase process $J$ if

$$
\left\|J_{n T+s}^{\pi}-q_{s}\right\| \rightarrow 0 \text { as } n \rightarrow \infty
$$

in total variation for all $s \in[0, T[$, independently from the initial distribution $\pi$.

Theorem 25. Let $(N, J)$ denote a periodic Markov-additive jump process with period $T$ and be $(N,+)$ a real vector space with base $\left(b_{i}: i \in I\right)$. Let the phase process $J$ have a periodic family of asymptotic distributions $\left(\pi_{t}: t \in[0, T[)\right.$. Define the mean rate vector during a period length by

$$
\lambda:=\left(\frac{1}{T} \int_{0}^{T} \int_{\Phi} d \pi_{t}(y) \int_{-\infty}^{\infty} c Q\left(t ; y, p r_{i}^{-1}(d c) \times \Phi\right) d t: i \in I\right)
$$

Assume that $\left\|p r_{i}\left(E\left(N_{t}\right)\right)\right\|<M(t)<\infty$ for all $i \in I$ and $t \in[0, T]$. Then

$$
\frac{N_{t}}{t} \rightarrow \lambda
$$

$P$-almost surely for all initial distributions.

Proof: Because of property 1 , the evolution of the process $(N, J)$ does only depend on the initial distribution $\mu$ of $J_{0}$ on the phase space $\Phi$. Since further every initial value $N_{0} \neq 0$ vanishes for $t \rightarrow \infty$, we can assume $N_{0}=0$ almost surely without loss of generality. Let $N_{t}^{\mu}$ denote the marginal process $N$ at time $t$ under initial phase distribution $\mu$. Further, let $\varphi_{t}=\mu P^{\Phi}(0, t)$ denote the distribution of the phase process at time $t$. 
Choose any $\varepsilon>0$. Then there is a number $k \in I N$ such that $\left\|\mu P^{\Phi}(0, n T)-\pi_{0}\right\|<$ $\varepsilon$ for all $n \geq k$ and the representation

$$
\begin{aligned}
\frac{N_{t}}{t} & =\frac{1}{t}\left(N_{k T}+\left(N_{\lfloor t / T\rfloor T}-N_{k T}\right)+\left(N_{t}-N_{\lfloor t / T\rfloor T}\right)\right) \\
& =\frac{1}{t} N_{k T}+\frac{1}{t} N_{(\lfloor t / T\rfloor-k) T}^{\varphi_{k T}}+\frac{1}{t}\left(N_{t}-N_{\lfloor t / T\rfloor T}\right)
\end{aligned}
$$

holds because of the periodicity of the process. Since the expectation kernel $E\left(N_{s}\right)$ is bounded for all $s \in \mathbb{R}_{0}$, the first and the last term will vanish for $t \rightarrow \infty$ almost surely. The second term equals

$$
\frac{1}{t} N_{(\lfloor t / T\rfloor-k) T}^{\varphi_{k T}}=\frac{1}{t} N_{(\lfloor t / T\rfloor-k) T}^{\pi_{0}}+\frac{1}{t} N_{(\lfloor t / T\rfloor-k) T}^{\varphi_{k T}-\pi_{0}}=\frac{1}{t} \sum_{m=k}^{\lfloor t / T\rfloor-1} N_{T}^{\pi_{0}}+\frac{1}{t} N_{(\lfloor t / T\rfloor-k) T}^{\varphi_{k T}-\pi_{0}}
$$

since $\pi_{0}=\pi_{0} P^{\Phi}(0, T)$ is invariant. The first term of this sum tends to

$$
\begin{aligned}
\lim _{t \rightarrow \infty} \frac{1}{t} \sum_{m=k}^{\lfloor t / T\rfloor-1} N_{T}^{\pi_{0}} & =\lim _{t \rightarrow \infty} \frac{(\lfloor t / T\rfloor-k) T}{t} \frac{1}{(\lfloor t / T\rfloor-k) T} \sum_{m=k+1}^{\lfloor t / T\rfloor} N_{T}^{\pi_{0}} \\
& =\lim _{t \rightarrow \infty} \frac{(\lfloor t / T\rfloor-k) T}{t} \cdot \lim _{n \rightarrow \infty} \frac{1}{(n-k) T} \sum_{m=k+1}^{n} N_{T}^{\pi_{0}} \\
& =\frac{1}{T} E\left(N_{T}^{\pi_{0}}\right)=\lambda
\end{aligned}
$$

almost surely, according to Kolmogorov's law of large numbers (cf. Bauer [2], p.86) and theorem 16. The assumption $\left\|\mu P^{\Phi}(0, k T)-\pi_{0}\right\|<\varepsilon$ implies

$$
P\left(N_{(\lfloor t / T\rfloor-k) T}^{\varphi_{k T}} \neq N_{(\lfloor t / T\rfloor-k) T}^{\pi_{0}}\right)<\varepsilon
$$

which means that

$$
N_{(\lfloor t / T\rfloor-k) T}^{\varphi_{k T}-\pi_{0}}=N_{(\lfloor t / T\rfloor-k) T}^{\varphi_{k T}}-N_{(\lfloor t / T\rfloor-k) T}^{\pi_{0}}=0
$$

with probability $1-\varepsilon$. Hence with probability $1-\varepsilon$, the convergence $\frac{N_{t}}{t} \rightarrow \lambda$ holds. Since $\varepsilon$ can be chosen arbitrarily small, there can be no set $N$ with $P(N)>0$ such that $\frac{N_{t}(\omega)}{\overbrace{}^{t}} \nrightarrow \lambda$ for all $\omega \in N$.

Remark 26. If there is a norm on $\Sigma$ (e.g. the supremum norm $\|x\|:=\sup _{i \in I}\left|p r_{i}(x)\right|$ for all $x \in \Sigma$ ) and $\Sigma$ is complete with respect to this norm, i.e. if $\Sigma$ is a Banach space, then the above theorem is valid for convergence in terms of the norm on $\Sigma$, too. This can be proven in exactly the same way by using the strong law of large numbers on Banach 
spaces (cf. Mourier [15] or Beck [3]) instead of Kolmogorov's law of large numbers, which is a statement for real-valued random variables.

Theorem 27. Let $(N, J)$ denote an homogeneous Markov-additive jump process and be $(N,+)$ a real vector space with base $\left(b_{i}: i \in I\right)$. Assume that the phase process $J$ has an asymptotic distribution $\pi$. Define the mean rate vector by

$$
\lambda:=\left(\int_{\Phi} d \pi(y) \int_{-\infty}^{\infty} c Q\left(y, p r_{i}^{-1}(d c) \times \Phi\right): i \in I\right)
$$

If $\lambda<\infty$, then

$$
\frac{N_{t}}{t} \rightarrow \lambda
$$

$P$-almost surely for all initial distributions.

Proof: This follows immediately from theorem 25, since an homogeneous process with asymptotic distribution $\pi$ is a periodic process with arbitrary period length $T>0$ and periodic family $\left(\pi_{t}=\pi: t \in[0, T[)\right.$ of asymptotic distributions. Furthermore,

$\int_{\Phi} d \pi(y) \int_{-\infty}^{\infty} c Q\left(y, p r_{i}^{-1}(d c) \times \Phi\right)=\frac{1}{T} \int_{0}^{T} \int_{\Phi} d \pi_{t}(y) \int_{-\infty}^{\infty} c Q\left(t ; y, p r_{i}^{-1}(d c) \times \Phi\right) d t$ holds for all $i \in I$, since $Q\left(t ; y, p r_{i}^{-1}(d c) \times \Phi\right)=Q\left(y, p r_{i}^{-1}(d c) \times \Phi\right)$ is constant in $t$. (-)

Remark 28. The statement of this theorem is the same as the result in Pacheco, Prabhu [19], theorem 6.17, if one specifies $\Sigma=I N_{0}^{r}$ and $\Phi$ being countable.

\section{References}

[1] E. Arjas, T.P. Speed (1973): "Topics in Markov-Additive Processes”, Math. Scandinav. 33, pp.171-192

[2] H. Bauer (1991): "Wahrscheinlichkeitstheorie", Walter de Gruyter (Berlin)

[3] A. Beck (1963): "On the strong law of large numbers", in: F. Wright: "Ergodic Theory", Academic Press (New York), pp.21-53

[4] N. Bourbaki (1969): "Intégration”, Diffusion C.C.L.S. (Paris)

[5] L. Breuer (2000): "Spatial Queues", Ph.D. thesis, University of Trier

[6] E. Çinlar (1972): "Markov Additive Processes I,II", Z. Wahrscheinlichkeitstheorie verw. Geb. 24, pp.85-93,95-121

[7] E. Çinlar (1975): "Lévy Systems of Markov Additive Processes", Z. Wahrscheinlichkeitstheorie verw. Geb. 31, pp.175-185 
[8] E. Çinlar (1977): "Markov Additive Processes and Semi-Regeneration”, Proc. 5th Conf. Probab. Theory, Brasov 1974, pp.33-49

[9] E. Çinlar (1982): "Regenerative systems and Markov additive processes", Seminar on Stochastic Processes in Evanston, Illinois, March 1982, Prog. Probab. Stat. 5, pp.123-147

[10] I.I. Ezhov, A.V. Skorokhod (1969): "Markov processes with homogeneous second component", Theory of Probability and its Applications 14, pp.1-13, 652-667

[11] I. Gikhman, A. Skorokhod (1969): "Introduction to the Theory of Random Processes", W.B. Saunders Company

[12] H. Herrlich (1986): "Topologie I: Topologische Räume”, Heldermann Verlag (Berlin)

[13] J. Hofmann (1998): "The BMAP/G/1 queue with level dependent arrivals", dissertation at the University of Trier, Dept. of Mathematics and Computer Science

[14] D. Lucantoni (1991): "New Results on the Single Server Queue with a Batch Markovian Arrival Process", Comm. Statist. - Stochastic Models, Bd. 7(1), pp.1-46

[15] E. Mourier (1953): "Elements Aleatoires dans un espace de Banach", Ann. Inst. H. Poincare 13, pp.161-244

[16] W. Nef (1977): "Lehrbuch der linearen Algebra”, Birkhäuser (Basel)

[17] J. Neveu (1961): "Une Generalisation des Processus a Acroissements Positifs Independants", Abh. math. Sem. Univ. Hamburg 25, pp.36-61

[18] P. Ney, E. Nummelin (1987): "Markov additive processes I,II", Ann. Probab. 15, pp.561592,593-609

[19] A. Pacheco, N.U. Prabhu (1995): "Markov-additive processes of arrivals", in: Dshalalow (ed.): "Advances in Queueing", CRC Press 